\title{
Genetic Diversity of 'Candidatus Liberibacter asiaticus' Revealed by Short Tandem Repeats and Prophage Typing Indicates Population Homogeneity in Brazil
}

\author{
Priscila Alves da Silva, ${ }^{1}$ Camila Giacomo Fassini, ${ }^{1}$ Laís Simões Sampaio, ${ }^{1}$ Gabriel Dequigiovanni, ${ }^{2}$ \\ Maria Imaculada Zucchi, ${ }^{2}$ and Nelson Arno Wulff ${ }^{1,3, \dagger}$
}

${ }^{1}$ Departamento de Pesquisa \& Desenvolvimento-Fundecitrus, Araraquara, SP, 14807-040, Brazil; ${ }^{2}$ Agência Paulista de Tecnologia dos Agronegócios, Polo Regional Centro-Sul, Rodovia SP 127, km 30, Piracicaba, SP, 13400-970 Brazil; and 3PPG Biotecnologia, IQ/UNESP Araraquara, SP, 14800-060, Brazil

Accepted for publication 25 January 2019.

\begin{abstract}
'Candidatus Liberibacter asiaticus' is the most common huanglongbingassociated bacteria, being present in Asia, South, Central, and North America. Genomic approaches enabled sequencing of ' $\mathrm{Ca}$. L. asiaticus' genomes, allowing for a broader assessment of its genetic variability with the application of polymerase chain reaction (PCR)-based tools such as microsatellite or short tandem repeat (STR) analysis. Although these tools contributed to a detailed analysis of strains from Japan, China, and the United States, Brazilian strains were analyzed in either too few samples with several STRs or in several strains with only a single microsatellite and a single PCR marker. We used 573 ' $\mathrm{Ca}$. L. asiaticus' strains, mainly collected from São Paulo State (SPS), in our genetic analyses, employing three STRs and several

prophage PCR markers. STR revealed a homogeneous population regardless of sampling year or geographic regions of SPS. Thirty-eight haplotypes were recognized with a predominance of VNTR_005 higher than 10 repeats, with VNTR_002 and VNTR_077 containing 11 and 8 repeats, respectively. This haplotype is indicated as class HE, which comprised $80.28 \%$ of strains. Classes HA and HB, predominant in Florida, were not found. A new genomic organization in the junction of prophages $\mathrm{SC} 2$ and $\mathrm{SC} 1$ is prevalent in Brazilian strains, indicating gene rearrangement and a widespread occurrence of a type 1 prophage as well as the presence of a type 2-like prophage. Our results indicate that ' $\mathrm{Ca}$. L. asiaticus' populations are homogeneous and harbor a new genomic organization in prophages type 1 and 2 .
\end{abstract}

Huanglongbing (HLB) is a century-old disease, currently of widespread occurrence due to the presence of 'Candidatus Liberibacter asiaticus' and its psyllid vector Diaphorina citri in main citriculture regions such as Brazil, China, and Asia in general; the United States; and Central America. Nevertheless, it has not been reported in Australia or in the Mediterranean Basin (Bové 2006; da Graça et al. 2016). Despite that, Trioza erytreae, another insect vector of Liberibacter, is threatening the Mediterranean Basin (Pérez-Otero et al. 2015). 'Ca. L. africanus' (Pietersen et al. 2010) and 'Ca. L. americanus' (Teixeira et al. 2005) are of limited geographic occurrence and, although ' $\mathrm{Ca}$. L. americanus' is almost eradicated in Brazil, the intraspecific variability of ' $\mathrm{Ca}$. L. africanus' is remarkable (Roberts et al. 2015, 2017). 'Ca. phytoplasma' spp., which are also phloem-limited, may similarly induce HLB symptoms (Chen et al. 2009; Teixeira et al. 2008; Wulff et al. 2019).

' $\mathrm{Ca}$. L. asiaticus' is subject of intense research related to genomics (Duan et al. 2009; Katoh et al. 2014; Lin et al. 2013; Tyler et al. 2009; Zhang et al. 2011; Zheng et al. 2014, 2018), which allowed the application of short tandem repeat (STR) analysis as a molecular diversity tool to study ' $\mathrm{Ca}$. L. asiaticus' populations (Chen et al. 2010; Islam et al. 2012; Katoh et al. 2011). Diversity

†Corresponding author: N. A. Wulff; E-mail: nelson.wulff@fundecitrus.com.br

Funding: Financial support was provided by São Paulo Research Foundation (FAPESP) (2015/07011-3) and a PD scholarship to P. A. da Silva (2016/01993-1). N. A. Wulff has a Conselho Nacional de Desenvolvimento Científico e Tecnológico (CNPq) fellowship (308997/2014-0). G. Dequigiovanni has a CNPq PD scholarship (150297/2018-1).

*The $\boldsymbol{e}$-Xtra logo stands for "electronic extra" and indicates that two supplementary figures and five supplementary tables are published online.

The author(s) declare no conflict of interest.

(C) 2019 The American Phytopathological Society studies of uncultured obligate pathogenic bacteria such as ' $\mathrm{Ca}$. L. asiaticus' are restricted to polymerase chain reaction (PCR) techniques, from size estimation of PCR products, sequence analysis of PCR-amplified genes, single-nucleotide polymorphism characterization, and PCR restriction fragment length polymorphism profiles to more broad applications such as microsatellite analysis and pan-genome analysis allowed by massive genome sequencing (Bastianel et al. 2005; Chen et al. 2010; Islam et al. 2012; Katoh et al. 2011, 2015; Matos et al. 2013; Tomimura et al. 2009; Wang et al. 2013; Zheng et al. 2016; Zhou et al. 2011). This is important to track possible introduction of ' $\mathrm{Ca}$. L. asiaticus' strains from country to country (Chen et al. 2010; Islam et al. 2012; Katoh et al. 2011), to understand the effect of either insect (Katoh et al. 2015) or plant (Matos et al. 2013) hosts on diversity evolution, and to address the intrinsic diversity of such pathogens. Furthermore, genomic studies of reference strains may take advantage of molecular studies to represent the most abundant or rare genotype in a certain time or region (Katoh et al. 2014; Zheng et al. 2018).

STR analysis identified in genomic sequences is largely used for population studies in bacteria (Gétaz et al. 2018; van Belkum et al. 2007), encompassing plant pathogens such as Xylella fastidiosa (Lin et al. 2015) and ' $C a$. L. asiaticus' populations (Chen et al. 2010; Islam et al. 2012; Katoh et al. 2011; Ma et al. 2014; Matos et al. 2013), including those from Brazil. ' $\mathrm{Ca}$. L. asiaticus' diversity was relatively uniform in these studies. However, either few strains were used for the STR analysis (Islam et al. 2012; Matos et al. 2013) or the analysis was restricted to two loci evaluated by PCR and sequence analysis (Deng et al. 2014), which may not depict the current molecular predominance of ' $\mathrm{Ca}$. L. asiaticus' population from Brazil along time and space. Although HLB was first described in Brazil 15 years ago (Coletta-Filho et al. 2004; Teixeira et al. 2005), its incidence level is rising slowly and currently is close to $18 \%$ (Fundecitrus 2018), mainly due to the application of a three-pronged management system (Bové 
2012). The objective of this study was to assess ' $\mathrm{Ca}$. L. asiaticus' strain diversity and genetic structure in a large sample population from Brazil, in regions where most of the intensive citriculture is located, covering distinct sampling times, geographic locations, and two host sources, to better assess the molecular variability of ' $C a$. L. asiaticus' strains by STR analysis as well as the presence and variation of prophages $\mathrm{SC} 1$ and $\mathrm{SC}$, enabling the comparison of our findings with related studies and a discussion of the importance of this characterization in the context of Liberibacter research.

\section{MATERIALS AND METHODS}

'Ca. L. asiaticus' populations. ' $C a$. L. asiaticus' samples comprised five subsets of populations: plant samples collected in 2010-11, specifically two samples from one municipality in the state of Minas Gerais and 151 samples from 47 municipalities in São Paulo State (SPS); plant samples collected between July and August 2015 in 42 municipalities in SPS; plant samples collected between 2014 and 2017 from a single farm in the municipality of Mogi Mirim, south of SPS; single ' $C a$. L. asiaticus'-infected $D$. citri individuals collected in 19 municipalities of SPS from July to August 2015, and nine ' $\mathrm{Ca}$. L. asiaticus' samples kept in greenhouse citrus plants. In total, 573 samples from Brazil were used (Supplementary Fig. S1). Eighty-two Florida ' $C a$. L. asiaticus' samples were collected in 2017 from Highlands, in central Florida (the United States) and received as DNA samples. ' $\mathrm{Ca}$. L. asiaticus' Poona (India) and Behai (China) strains were received as DNA extracts obtained from INRA/Bordeaux (France). Every sample was from a single tree or a single psyllid.

Samples from Brazil had DNA extracted at the Fundecitrus Diagnostic Service (Murray and Thompson 1980). DNA was quantified in spectrophotometer (NanoDrop; ThermoFisher Scientific, Waltham, MA, U.S.A.) and the presence of ' $\mathrm{Ca}$. L. asiaticus' was detected by either conventional (Hocquellet et al. 1999) or quantitative (Li et al. 2006) PCR.

Multilocus STR analysis. Three STR loci were used for ' $\mathrm{Ca}$. L. asiaticus' diversity analysis. These loci showed polymorphism in previous ' $C a$. L. asiaticus' studies (Chen et al. 2010; Islam et al. 2012; Katoh et al. 2011; Matos et al. 2013) containing variable number of tandem repeats (VNTR) for motifs AGACACA (Chen et al. 2010; Islam et al. 2012; Katoh et al. 2011; Matos et al. 2013), CAGT, and TTTG (Islam et al. 2012; Katoh et al. 2011; Matos et al. 2013) (Table 1). Genotyping was performed with fluorophoremarked primers and PCR was carried out (MasterCycler gradient; Eppendorf, Hamburg, Germany) with a single step of 4 min at $94^{\circ} \mathrm{C}$; followed by 30 cycles of $30 \mathrm{~s}$ at $94^{\circ} \mathrm{C}, 45 \mathrm{~s}$ at $55^{\circ} \mathrm{C}$, and $30 \mathrm{~s}$ at $72^{\circ} \mathrm{C}$; and a final elongation step of $20 \mathrm{~min}$ at $72^{\circ} \mathrm{C}$. Resolution of PCRamplified fragments was assessed in a capillary sequencer (ABI3730XL; Applied Biosystems, Foster City, CA, U.S.A.) and peak detection and analysis were done with GeneMapper (version 4.01; Applied Biosystems) at CREBIO, UNESP-FCAV (Jaboticabal, SP, Brazil). Internal Lane Standard 60 to 600 bp, ROX (Norgen Biotek Corp., Thorold, ON, Canada) was used as the DNA ladder. STR analysis was carried out for all Brazilian samples.
Where indicated, PCR amplicons for loci VNTR_005/trn1 (motif A) (Chen et al. 2010), VNTR_002 (motif C), and VNTR_077 (motif D) (Katoh et al. 2011) were directly sequenced with reverse primer using chain terminator sequencing (Sanger sequencing). Sequence reads were analyzed with the software CodonCodeAligner (version 8.0.1; CodonCode Corporation, Centerville, MA, U.S.A.), automatically clipped at ends, checked manually for quality, and assembled into contigs. Consensus sequences were manually curated and a quality cut-off of 20 was used to edit the consensus. Repeat number for each STR was counted manually. Values generated from reference samples were used as calibrators for repeat counting derived from genotyping with STR-fluorophoremarked primers. Locus VNTR_005 amplification with primers LapGP-1r/LapGP-1r (Chen et al. 2010) and amplicon sequencing allowed the identification of binding sites for LasSSR-E-f/LasSSRE-r primers (Islam et al. 2012).

The presence and variation of VNTR_005 were explored in the ' $C a$. L. asiaticus' population from $2010-11$ by both STR analysis (Islam et al. 2012) and amplicon sequencing (Chen et al. 2010; Ma et al. 2014). PCR amplicons from primer set LapGP-1f/LapGP-1r (Chen et al. 2010) were sequenced with primers Las_F1 (TACAA ATGAAACAATCTGCC) and Las_F2 (GCTTGATCTACCATTT TCCG) and motif AGACACA was manually counted and compared with STR analysis data.

Differences in means between haplotype classes within population subsets were compared by Fisher's exact test $(P<0.05)$, with the statistical software BioEstat, version 5.0 (Ayres et al. 2007).

Genetic diversity using STR. Haplotypes were identified based on allelic combination of multilocus STR. A haplotype or haploid genotype is the combination of alleles found at two or more loci in a single individual. Clone-corrected data were generated by removing repeated genotypes (Islam et al. 2012). For the subsets of populations (2010-11 samples, 2015 samples, samples from a single farm in Mogi Mirim, and samples from the 2015 Asian citrus psyllid [ACP]), samples were reclassified according to the geographic region where each sample was collected: center, northwest, north, southwest and south of SPS. These regions follow the historic division of HLB surveys carried out by Fundecitrus (https://www.fundecitrus.com.br/ levantamentos/greening). ' $\mathrm{Ca}$. L. asiaticus' strains from greenhouse citrus plants were removed from this particular analysis due to their low sample size. Samples with double peaks in a single locus in STR analysis were excluded, because prokaryotes are considered haploid individuals. These samples were considered as having more than a single ' $C a$. L. asiaticus' strain. Therefore, the remaining 470 strains were subsequently analyzed.

Genetic diversity revealed by STR was described by estimating the number of multilocus genotypes, total number of alleles, number of private alleles, and Nei's diversity index. Estimations were obtained with GenAlEx v. 6.5 (Peakall and Smouse 2012). Private alleles are found only in a single population among a broader collection of populations (Szpiech and Rosenberg 2011).

Hierarchical distribution of genetic variation within and among groups was evaluated using locus-by-locus analysis of molecular

TABLE 1. Primers for short tandem repeat (STR) amplification and characterization of three loci in the genome of 'Candidatus Liberibacter asiaticus' strains

\begin{tabular}{lcllc}
\hline Locus $^{\mathrm{v}}$ & Motif $^{\mathrm{w}}$ & \multicolumn{1}{c}{ STR $^{\mathrm{x}}$} & \multicolumn{1}{c}{ Primer sequences $^{\mathrm{y}}$} & Genome position $^{\mathrm{z}}$ \\
\hline VNTR_002 & $\mathrm{C}$ & $(\text { CAGT })_{8}$ & 002_f TAMRA-TGATAATATAGAAAGAGGCGAAGC & $537,540-537,563$ \\
& & & 002_r TCCATACCCAAAAGAAAAGCA & Katoh et al. 2011 \\
VNTR_005 & A & $(\text { AGACACA })_{5}$ & LasSSR-E-f FAM-GATCAGTAGTCTATCACCAC & $354,227-354,246$ \\
& & & LasSSR-E-r TACTGGAAACAAATGGAATAC & $354,805-354,824$ \\
VNTR_077 & D & \multirow{2}{*}{$(\text { TTTG })_{14}$} & 077_f JOE-TGACTGATGGCAAAAGATGG et al. 2012 & $655,220-655,239$ \\
& & & 077_r AGACACGCCAAACAAGGAAT & Katoh et al. 2011 \\
\hline
\end{tabular}

v Loci nomenclature according to Katoh et al. (2011).

${ }^{w}$ Motif names as proposed by Matos et al. (2013).

x Nucleotide sequence and number of tandem repeat in reference genome Las psy62 (Chen et al. 2010; Duan et al. 2009; Katoh et al. 2011).

y Primer names, sequences and $5^{\prime}$-fluorophore in forward primer.

z Reference genome Las psy62 NC_012985 (Duan et al. 2009) version 30/03/2017. 
variance (AMOVA) with GenAlEx v. 6.5 (Peakall and Smouse 2012). Significance was assessed with 9,999 permutations.

Genetic structure based on STR variation was evaluated with discriminant analysis of principal components (DAPC) (Jombart et al. 2010) with adegenet 2.0.0 (Jombart and Ahmed 2011) for R (R Core Team 2015). This methodology does not have requirements such as absence of linkage and Hardy-Weinberg equilibrium within clusters (Jombart et al. 2010). DAPC summarizes the maximum variation between groups while minimizing within-group differences (Roullier et al. 2013). The K-means algorithm for clustering of principal components, based on the Bayesian Information Criterion, was computed to assess the best number of clusters.

The dispersion of genetic diversity among populations and strains was evaluated by principal component analysis (PCA), carried out with the R Statistical Software, ade4 package (Dray and Dufour 2007).

Genetic relationships and divergence among individuals and groups of strains were investigated with an arithmetic mean dendrogram (unweighted pair-group method with arithmetic mean [UPGMA]) based on Nei's genetic distance (Nei 1972) obtained with poppr (Kamvar et al. 2014) for R (R Core Team 2015). To infer the confidence of relationships, 10,000 bootstrap replicates were performed. Final trees were formatted in FigTree 1.4.1 (http:// tree.bio.ed.ac.uk/software/figtree/) and MEGA, version 7 (Kumar et al. 2016).

A minimum spanning network (MSN) was constructed with poppr (Kamvar et al. 2014) for $\mathrm{R}$ ( $\mathrm{R}$ Core Team 2015) to assess relationships among haplotypes, using the Bruvo's genetic distance (Bruvo et al. 2004), which is recommended for STR loci.

Presence of prophages SC1 (type 1) and SC2 (type 2). PCR products specific for prophages type 1 and 2 (Table 2) (Zheng et al. 2016) were amplified for the nine reference samples, using the Go Taq Flexi DNA polymerase according to manufacturer's instructions (Promega Corporation, Madison, WI, U.S.A.), and directly sequenced with amplification primers. PCR product SC1-7 was additionally sequence with internal primers 448-R (TATAG GCGGGTTAATGGGCG), 578-F (AAGATCTACTCATTTGTCC CCC) and 898-F (TCGAATACCCCTCGTCCAAC). Primer sets SC1-7F/SC1-7R and SC2-8F/SC2-8R amplified sizes different than reported (Zheng et al. 2016) and partial consensus sequences were further analyzed by BLASTn and BLASTp (https://blast.ncbi.nlm. nih.gov/Blast.cgi).

The presence of the hyvI and hyvII loci (Table 2) (Zhou et al. 2011) was evaluated by conventional PCR using the Phusion High-

TABLE 2. Primers used to assess the presence prophage SC1 (type 1) and prophage SC2 (type 2) in 'Candidatus Liberibacter asiaticus' population

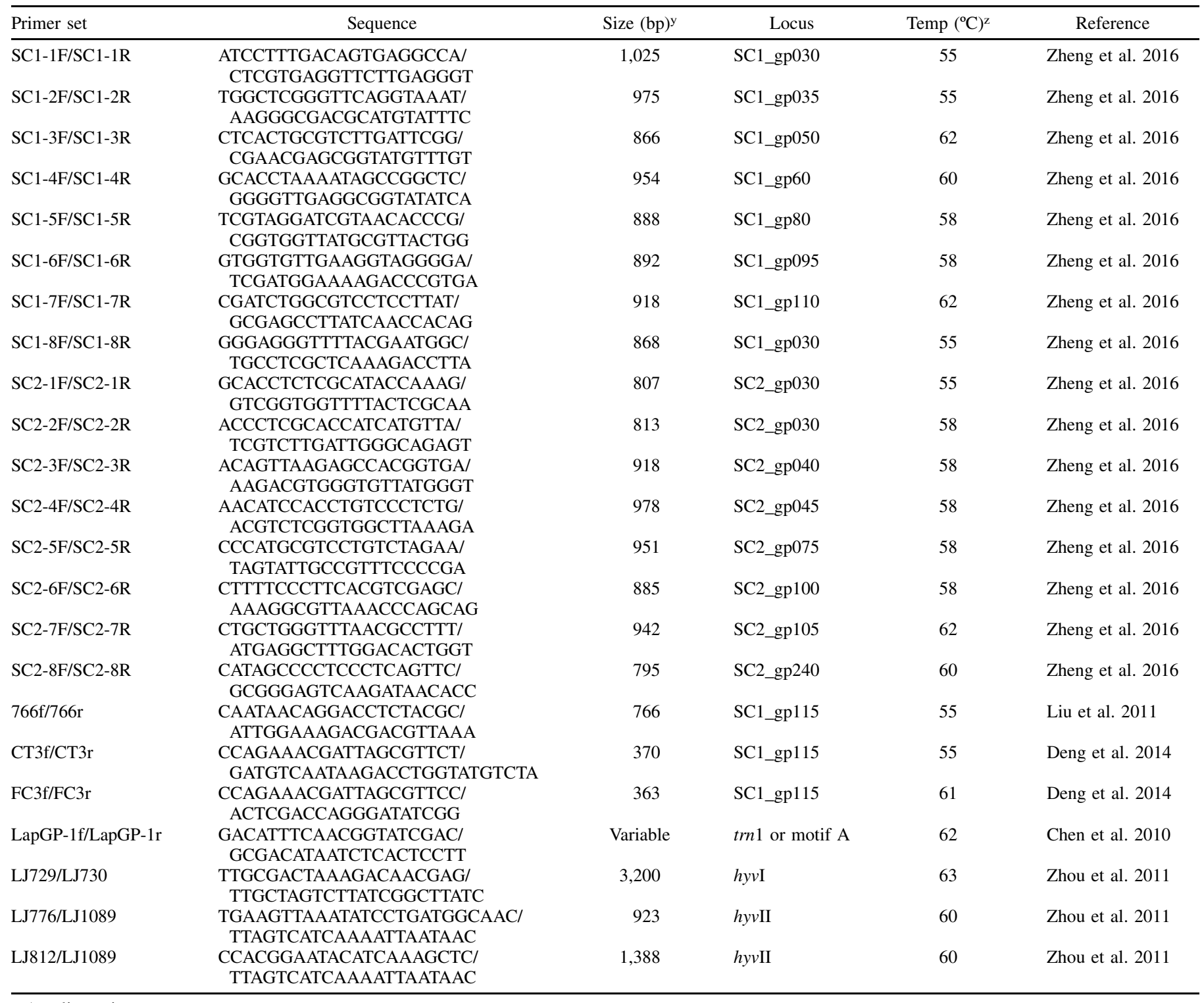

y Amplicon size.

z Temperature at which annealing stage was carried out during polymerase chain reaction. 
Fidelity DNA polymerase according to the manufacturer's instructions (ThermoFisher Scientific) in the ' $\mathrm{Ca}$. L. asiaticus' population from 2010-11.

The ' $\mathrm{Ca}$. L. asiaticus' population collected in 2015 was screened for the presence of prophages SC1 (type 1) and SC2 (type 2) using two PCR markers specific for each prophage (SC1-1F/SC1-1R and $\mathrm{SC} 1-4 \mathrm{~F} / \mathrm{SC} 1-4 \mathrm{R}$ in the case of prophage type 1; SC2-1F/SC2-1R and SC2-8F/SC2-8R in the case of prophage type 2) (Zheng et al. 2016). This analysis employed duplex PCR with SC1-4/SC2-8 and SC1-1/SC2-1, using Go Taq Flexi DNA polymerase. In the case of prophage type 1, a third locus was also used: PCR primer sets CT3f/ CT3r and FC3f/FC3r (Table 2), which are able to detect the large subunit of the phage terminase and to characterize strains as Term-A or Term-G (Deng et al. 2014). Eventually, amplification and sequencing of locus 766 with primer set $766 f / 766$ r (Table 2 ) and primers 766_FF (CCATAACGAGCTATGATCCC) and 766_RR (ATACGTCCTACACATCGTCG) were also carried out (Liu et al. 2011).

Sequence analysis, annotation, and sequence comparisons of prophage genes. The PCR product obtained with primer pair SC2-8F/SC1-1R for sample 9PA was directly sequenced (Supplementary Table S1). Sequence analysis was carried out in CodonCodeAligner. GeneMark was used for open reading frame (ORF) prediction (Besemer and Borodovsky 1999) (http://exon. gatech.edu/GeneMark/) and protein annotation was performed after BLASTp comparison. ' $\mathrm{Ca}$. L. asiaticus' genome and prophage sequences were downloaded from the National Center for Biotechnology Information. Sequence alignments were created with Clustal Omega (Sievers and Higgins 2018) (https://www.ebi.ac.uk/ Tools/msa/clustalo/).

Based on the gene order and sequence of this 6.1-kbp PCR product, PCR primer 9PA-1F (CGTCAAAGGGGAAATTGGAAAG) and 9PA-1R (GTATAGAAGTCGCAAATGTACG) yielding a 567-bp fragment were designed to test the occurrence of this mosaic locus in ' $\mathrm{Ca}$. L. asiaticus' samples, in that 414 samples from SPS (Brazil) and 82 samples from Highlands, central Florida were tested.

\section{RESULTS}

' $C a$. L. asiaticus' strains were detected in plant and psyllid samples by PCR tests. After a preliminary STR typing analysis in haplotype classes, ' $C a$. L. asiaticus' samples were used for genetic analysis.

STR analysis. Nine ' $\mathrm{Ca}$. L. asiaticus' reference strains were assessed for the three STRs by amplicon sequencing and manual count of repeats as well as by capillary electrophoresis and repeat estimation based on amplicon size. Although sequencing always produced a single number of repeats and the capillary electrophoresis-based detection matched this value, the latter technique allowed detection of two peaks for some samples, called double peaks hereafter. That was the case for sample 4PA in VNTR_002, with 10 and 11 repeats of CAGT (motif C), while VNTR_005 had double peaks for sequence AGACACA (motif A) in samples 3PA, 4PA, and 7PA, with a combination of 14, 15, and 16 repeats, respectively (Supplementary Table S2).

The number of repeats in VNTR_005 was determined by PCR product sequencing and manual count, in addition to being estimated from the size of the fluorophore-based detection of amplified product for the ' $\mathrm{Ca}$. L. asiaticus' population subset 2010-11. The number of repeats for motif A was the same for 130 of 139 samples tested by both techniques. The remaining nine samples had a single count in the number of repeats in sequencing data, although double peaks were detected in the fluorophore-based technique, which was the same situation described for reference strains 3PA, 4PA, and 7PA.

STR analysis was carried out for 573 samples composed of ' $\mathrm{Ca}$. L. asiaticus' strains in plant and psyllid hosts. Overall, 476 samples had a single peak for each locus, while 97 had double peaks for one, two, or three loci (Supplementary Table S3). All three STRs were polymorphic, totaling 38 haplotypes within a population of 470 strains (Supplementary Table S5).

VNTR_005 was found in the range of 6 to 18 repeats, with 15 repetitions being the most frequent (289 samples). Seventy-nine samples had double peaks for this locus, indicating the presence of two sequences with distinct sizes, in that sequences with 15 repetitions (49 of 79) were also the most frequent. Double peaks for this STR were confirmed in gel electrophoresis for reference strains, although doublet bands were not always observed in agarose gel electrophoresis, particularly in the occurrence of a single repeat difference (data not shown). In total, 338 of 573 samples had 15 repetitions for this motif (59\% of strains). VNTR_002 (CATG) was found in the range of 9 to 14 repeats, with 11 repeats being the most frequent (500 of 573). In all, 14 samples had double peaks for this locus, with 13 of 14 samples having 11 repeats. VNTR_077 (TTTG) was found in the range of 5 to 11 repeats, with 8 repeats being the most common (537 of 573). Fourteen samples had double peaks for this locus, all of them including eight repeat motifs. The most frequent haplotype, found in 233 samples, had 15 repetitions at VNTR_005, 11 repetitions at VNTR_002, and 8 repetitions at VNTR_077 (Table 3).

' $C a$. L. asiaticus' strains were grouped in haplotype classes according to the STR in the three loci (Table 3), following a scheme

TABLE 3. 'Candidatus Liberibacter asiaticus' haplotypes classes based on the short tandem repeat (STR) analyses found in loci VNTR_005, VNTR_002, and VNTR_077y

\begin{tabular}{|c|c|c|c|c|c|c|c|}
\hline \multirow[b]{2}{*}{ Class $^{\mathrm{z}}$} & \multirow{2}{*}{$\frac{\text { Motif A }}{\text { VNTR_005 }}$} & \multicolumn{2}{|c|}{ Motif C } & \multicolumn{2}{|c|}{ Motif D } & \multirow[b]{2}{*}{$N$} & \multirow[b]{2}{*}{ Reference } \\
\hline & & Size (bp) & VNTR_002 & Size (bp) & VNTR_077 & & \\
\hline HA & $<10$ & 509 & 8 & 527 & 14 & $\mathrm{nf}$ & Matos et al. 2013 \\
\hline $\mathrm{HB}$ & $>10$ & 513 & 9 & 503 & 7 & $\mathrm{nf}$ & Matos et al. 2013 \\
\hline HD & $>10$ & 521 & 11 & 503 & 7 & 7 & This study \\
\hline $\mathrm{HE}$ & $>10$ & 521 & 11 & 507 & 8 & 460 & This study \\
\hline $\mathrm{HF}$ & $>10$ & 521 & 11 & 511 & 9 & 6 & This study \\
\hline $\mathrm{HI}$ & $>10$ & 529 & 13 & 507 & 8 & 6 & This study \\
\hline HJ & $<10$ & 521 & 11 & 507 & 8 & 5 & This study \\
\hline Misc & $>10$ & $513,517,521,529$ & $9,10,11,13$ & $495,503,507,511$ & $5,7,8,9$ & 4 & This study \\
\hline
\end{tabular}

y Motifs A, C, and D according to Matos et al. (2013), based on markers from Chen et al. (2010) and Katoh et al. (2011). STR was calibrated with reference strains, where amplicon was sequenced and number of repeats manually curated. Matos et al. (2013) reported 8 repeats for motif D in a 503 bp amplicon. In our analysis, polymerase chain reaction products where 8 STR were found in motif D, produced a single peak with $507 \mathrm{bp}$ in the fluorophore-based detection, value used for normalization. Double STR > 10 in motif A, STR 11 in motif C and STR 8 in motif D had 58 samples classified as haplotype class HE. Additionally, STR with double peaks for one, two or three motifs comprised 39 samples, not classified. Size $=$ amplicon size, $N=$ number of strains, and $n f=$ not found.

${ }^{\mathrm{z}}$ Haplotype class. Miscellaneous group (Misc) has samples of a single STR combination for VNTR_002 and VNTR_077. 
used for ' $\mathrm{Ca}$. L. asiaticus' strains from Florida (Matos et al. 2013). This classification first considers the repeat motif for locus VNTR_005 as lower or higher than 10 (Chen et al. 2010; Ma et al. 2014). Second, the repeat number in the less variable VNTR_002 and VNTR_077 (Matos et al. 2013) is considered. Brazilian ' $\mathrm{Ca}$. L. asiaticus' strains were grouped into eight classes (Table 3): the main haplotype class was represented by 460 strains (HE) or $80.28 \%$ of the samples, followed by 34 samples in class HC. Additional classes ranged from three to nine samples (classes HD, $\mathrm{HF}, \mathrm{HG}, \mathrm{HH}, \mathrm{HI}$, and HJ). A miscellaneous group contained four samples of a single STR combination for VNTR_002 and VNTR_077. Among haplotype classes present in Brazil, only class HJ had VNTR_005 with less than 10 repeats (Table 3). In addition to having a single peak for each of the locus (233 strains or $40.66 \%$ of the samples), class HE contains 58 strains with double peaks for VNTR_005, both higher than 10 repeats. VNTR_002 and VNTR_077 contain 11 and 8 repeats, respectively (Table 3).

Further information collected from these 573 samples includes the evolution of haplotype classes along time and space, because these strains belonged to four subsets of populations, in addition to the ' $C a$. L. asiaticus' reference strains kept in a greenhouse (Table 4). In every subset, the haplotype class HE was significantly predominant. The subset of samples collected in a single orchard between October 2014 and January 2017 (on Mogi Mirim farm) had the lowest percentage of class HE among plant samples, with a concomitant increase in class HC. Single psyllid samples collected between July and August 2015 followed the same trend of frequency of classes found in plants but had a higher presence of double alleles per locus (Table 4). The subset of nine strains kept in a greenhouse was class HE, with ' $\mathrm{Ca}$. L. asiaticus' strain 4PA having double peaks for motifs $\mathrm{A}$ and $\mathrm{C}$ and, therefore, being left out of the classification.

Double peaks in a single locus were not rare is our population study (97 samples or $16.9 \%$ of the population), accounting for 8.5 , 20.7 , 4.7, and $24.6 \%$ of ' $\mathrm{Ca}$. L. asiaticus' strains from 2010-11 subset, 2015 subset, Mogi Mirim farm subset, and ACP subset, respectively. Except for ' $\mathrm{C} a$. L. asiaticus' strains with double peaks at VNTR_005 and classified as HE (58 samples), the remaining strains with this feature were not assigned a haplotype class because there was a great variation among samples, leaving few samples for each haplotype.

We observed that STR locus count by genotyping is more reliable than sequencing, showing a higher number of double peaks in STR. In the case of VNTR_005, samples with double peaks were confirmed by gel electrophoresis (data not shown). The intrinsic difficulty of sequencing repetitive regions is also a concern.

Genetic diversity analysis. In the various populations of ' $\mathrm{Ca}$. L. asiaticus', the number of alleles per locus varied from 1 to 10 (Table 5). Locus VNTR_005 presented a higher variability with a higher number of alleles (Na) found (12 alleles), having an overall Nei's genetic diversity of 0.619 (Table 5). Loci VNTR_002 and
VNTR_077 had each five alleles (Table 5; Supplementary Table S4).

Private alleles were found in population 5 , corresponding to alleles with 6, 8, and 10 repetitions at locus VNTR_005 and 9 repetitions at locus VNTR_002, all with a frequency of 0.008 . In population 7, only one private allele of nine repetitions at locus VNTR_005 was found, with a frequency of 0.056 . In population 4 , a private allele was also found, which corresponded to five repetitions at locus VNTR_077, with a frequency of 0.038 (Table 5).

Genetic diversity by locus ranged from 0 to 0.72 in the several populations. Mean genetic diversity among populations was low, ranging from 0.204 (population 9) to 0.349 (population 6). Populations $1,3,6,7$, and 8 showed a greater genetic diversity at locus VNTR_005. Population 2, from northwest of SPS, was the only one to have the greatest genetic diversity at locus VNTR_002 instead of VNTR_005. Nei's genetic diversity in the whole area (all strains) was higher for locus VNTR_005, lower for locus VNTR_002, and the lowest for locus VNTR_077 (Table 5).

The highest allele number per locus among populations was related to the highest allele number present in locus VNTR_005 (motif A). 'Ca. L. asiaticus' strains from plant samples collected in 2010 to 2015 in the south region (population 5) had the highest number of alleles (average of six alleles per locus). VNTR_005 had 10 alleles, with 3 private alleles, while VNTR_002 and VNTR_077 had 4 alleles, with VNTR_002 having 1 private allele. Next came the center region (population 1, with 5.33 alleles per locus and 8 alleles for VNTR_005) and then the north region (population 3, with 4.67 alleles per locus and 7 alleles for VNTR_005). Population 6, comprising samples collected on a single farm in the municipality of Mogi Mirim, presented a mean of four alleles per locus and genetic diversity estimate of 0.349 , representing the local variability found in a single location (Table 5).

Genetic structure. UPGMA clustering analysis of ' $\mathrm{Ca}$. L. asiaticus' populations showed that bootstrap values were low, indicating unsupported clusters (Fig. 1), except for the population clusters 3 and 8 from the north region, with a bootstrap value of 70 . UPGMA clustering analysis of ' $C a$. L. asiaticus' strains, with a clone-corrected dataset from each population, confirmed the absence of reliable population structure (Supplementary Fig. S2). A DAPC analysis reinforced the absence of structure among strains studied (Fig. 2). Lack of structure among ' $\mathrm{Ca}$. L. asiaticus' populations was confirmed with descriptive data from AMOVA (Table 6) and PCA (data not shown). Comparative results among nine populations showed that these populations did not have significant genetic variation (Table 6). The AMOVA result for relationship comparison showed no significant genetic variation among regions (center, northwest, north, southwest, and south) (Table 6). In general, AMOVA and statistic estimates $\Phi_{\mathrm{ST}}$ confirmed low levels of genetic structure, showing that diversity was concentrated within groups. A slight yet significant variation was obtained only among sampling times, highlighting the fact that

TABLE 4. 'Candidatus Liberibacter asiaticus' population subsets and percentage of haplotype classes based on short tandem repeats (STRs) found in loci VNTR_005, VNTR_002, and VNTR_077y

\begin{tabular}{|c|c|c|c|c|c|c|}
\hline Haplotype class ${ }^{\mathrm{z}}$ & 2010-11 & 2015 & Mogi Mirim farm & Psyllids 2015 & Reference strains & Overall \\
\hline $\mathrm{HC}$ & $6.5 \mathrm{~b}$ & $4.7 \mathrm{c}$ & $14.0 \mathrm{~b}$ & $5.8 \mathrm{bc}$ & $\ldots$ & $5.93 \mathrm{~b}$ \\
\hline HD & $1.3 \mathrm{~cd}$ & $1.3 \mathrm{~d}$ & $2.3 \mathrm{bc}$ & 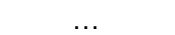 & $\ldots$ & $1.22 \mathrm{c}$ \\
\hline $\mathrm{HE}$ & $81.7 \mathrm{a}$ & $81.6 \mathrm{a}$ & $76.7 \mathrm{a}$ & $72.5 \mathrm{a}$ & 88.9 a & $80.28 \mathrm{a}$ \\
\hline HG & $0.7 \mathrm{~cd}$ & $0.7 \mathrm{~d}$ & $\ldots$ & $\ldots$ & $\ldots$ & $0.52 \mathrm{c}$ \\
\hline $\mathrm{HH}$ & $0.7 \mathrm{~cd}$ & $1.3 \mathrm{~d}$ & $4.7 \mathrm{bc}$ & $2.9 \mathrm{bc}$ & $\ldots$ & $1.57 \mathrm{c}$ \\
\hline HI & $2.0 \mathrm{bcd}$ & $0.7 \mathrm{~d}$ & $\ldots$ & $1.4 \mathrm{c}$ & $\ldots$ & $1.05 \mathrm{c}$ \\
\hline $\mathrm{HJ}$ & $1.3 \mathrm{~cd}$ & $0.3 \mathrm{~d}$ & $\ldots$ & $2.9 \mathrm{bc}$ & $\ldots$ & $0.87 \mathrm{c}$ \\
\hline Number of samples & 153 & 299 & 43 & 69 & 9 & 573 \\
\hline
\end{tabular}

y Mean values in each column followed by different lowercase letter are significantly different when compared with Fisher's exact test $(P<0.05)$.

${ }^{\mathrm{z}}$ STR with double peaks for one, two, or three motifs comprised 39 samples, not grouped in classes. Class HE contains double STR $>10$ for locus VNTR_005. 
populations were quite similar and explaining $99 \%$ of the total genetic variation (Table 6).

The presence of 38 distinct haplotypes among 470 strains with a single peak from the total population was verified through genealogical analysis performed by MSN (Fig. 3). The most frequent genotype found in the populations corresponded to haplotype class HE (Tables 3 and 4).

Prophage survey and analysis. ' $C a$. L. asiaticus' strains were further analyzed for the presence of prophage genes by using PCR. Although hyvII was not detected in our ' $\mathrm{Ca}$. L . asiaticus' population subset 2010-11, hyvI was PCR amplified in 127 strains (data not shown). In six strains, double bands were present and 14 samples did not amplify. We observed variation in the size of the amplicon, with the presence of four distinct sizes between 1.8 and $2.6 \mathrm{kbp}$. PCR products from type 1 and 2 prophages from reference ' $\mathrm{Ca}$. L. asiaticus' strains showed an identical amplification profile among nine samples. Although the eight PCR markers for prophage type 1 were amplified (Fig. 4), only PCR set SC2-8F/SC2-8R, out of the eight PCR markers for prophage type 2, did amplify (data not shown

TABLE 5. Estimates of genetic diversity for the short tandem repeat loci of 'Candidatus Liberibacter asiaticus' strains from Brazil ${ }^{\mathrm{z}}$

\begin{tabular}{|c|c|c|c|}
\hline Population, locus & $N$ & $\mathrm{Na}$ & $h$ \\
\hline \multicolumn{4}{|c|}{ 1: 2010 to 2015 center } \\
\hline VNTR_005 & 183 & 8 & 0.664 \\
\hline VNTR_002 & 183 & 4 & 0.192 \\
\hline VNTR_077 & 183 & 4 & 0.085 \\
\hline Mean & 183 & 5.33 & 0.314 \\
\hline \multicolumn{4}{|c|}{ 2: 2010 to 2015 northwest } \\
\hline VNTR 005 & 8 & 2 & 0.375 \\
\hline VNTR_002 & 8 & 3 & 0.531 \\
\hline VNTR_077 & 8 & 1 & 0 \\
\hline Mean & 8 & 2 & 0.302 \\
\hline \multicolumn{4}{|c|}{ 3: 2010 to 2015 north } \\
\hline VNTR_005 & 34 & 7 & 0.711 \\
\hline VNTR_002 & 34 & 3 & 0.112 \\
\hline VNTR_077 & 34 & 4 & 0.166 \\
\hline Mean & 34 & 4.67 & 0.33 \\
\hline \multicolumn{4}{|c|}{ 4: 2010 to 2015 southwest } \\
\hline VNTR_005 & 26 & 4 & 0.512 \\
\hline VNTR_002 & 26 & 2 & 0.074 \\
\hline VNTR_077 & 26 & $3(1)$ & 0.145 \\
\hline Mean & 26 & 3 & 0.244 \\
\hline \multicolumn{4}{|c|}{ 5: 2010 to 2015 south } \\
\hline VNTR_005 & 126 & $10(3)$ & 0.498 \\
\hline VNTR 002 & 126 & $4(1)$ & 0.175 \\
\hline VNTR_077 & 126 & 4 & 0.047 \\
\hline Mean & 126 & 6 & 0.24 \\
\hline \multicolumn{4}{|c|}{ 6: Mogi Mirim Farm south } \\
\hline VNTR_005 & 41 & 6 & 0.623 \\
\hline VNTR_002 & 41 & 3 & 0.328 \\
\hline VNTR_077 & 41 & 3 & 0.094 \\
\hline Mean & 41 & 4 & 0.349 \\
\hline \multicolumn{4}{|c|}{ 7: Psyllids 2015 center } \\
\hline VNTR_005 & 36 & $7(1)$ & 0.691 \\
\hline VNTR_002 & 36 & 4 & 0.295 \\
\hline VNTR_077 & 36 & 1 & 0 \\
\hline Mean & 36 & 4 & 0.329 \\
\hline \multicolumn{4}{|c|}{ 8: Psyllids 2015 north } \\
\hline VNTR_005 & 5 & 4 & 0.72 \\
\hline VNTR_002 & 5 & 1 & 0 \\
\hline VNTR_077 & 5 & 2 & 0.32 \\
\hline Mean & 5 & 2.33 & 0.347 \\
\hline \multicolumn{4}{|c|}{ 9: Psyllids 2015 southwest } \\
\hline VNTR_005 & 11 & 4 & 0.446 \\
\hline VNTR_002 & 11 & 2 & 0.165 \\
\hline VNTR_077 & 11 & 1 & 0 \\
\hline Mean & 11 & 2.33 & 0.204 \\
\hline \multicolumn{4}{|l|}{ Overall } \\
\hline VNTR_005 & 470 & 12 & 0.619 \\
\hline VNTR_002 & 470 & 5 & 0.203 \\
\hline VNTR_077 & 470 & 5 & 0.079 \\
\hline
\end{tabular}

${ }^{\mathrm{z}} \mathrm{N}=$ number of individuals, $\mathrm{Na}=$ number of alleles (number of private alleles), and $h=$ Nei's genetic diversity. for the SC2 type). The same pattern was observed for the Poona strain from India (type 1 prophage), whereas the Behai strain from China had an opposite pattern (type 2 prophage) (data not shown). Three DNA samples from central Florida amplified with both prophage type 1 and 2 primers (data not shown).

The ' $C a$. L. asiaticus' 2015 population screened for prophage markers was the same used for STR analysis. PCR with primers CT3f/CT3r, located in prophage SC1 (Deng et al. 2014), amplified the expected DNA size for 297 of 299 samples, in that 2 samples remained without amplification. However, these two samples were positive in PCR 766f/766r, indicating the presence of SC1_gp115 in all samples (Liu et al. 2011), but also negative in PCR FC3f/FC3r. In fact, none of the samples did amplify with PCR FC3f/FC3r, indicating the absence of Term-G type (Deng et al. 2014). Thus, all samples, except for two, were of the large subunit phage terminase type Term-A.

Of 299 strains, 289 amplified for PCR markers in prophage SC1 as well as for SC2-8F/SC2-8R. PCR marker SC2-1F/SC2-1R did not amplify in any sample from Brazil. In eight samples, one of the PCR markers failed in the amplification for type 1 or type 2 prophages. Two samples failed in all PCR markers, except for and only for 766f/766r and CT3f/CT3r. The ' $C a$. L. asiaticus' field population from 2015 is very homogeneous for prophage markers, in that $96.7 \%$ have an identical profile and seem to have a complete SC1-type prophage, similar to SC1 (Zhang et al. 2011), and a partial SC2-type prophage, more likely being an SC2-like prophage. Complete absence of prophages type 1 and 2 was not recorded.

PCR with primer set SC1-7F/SC1-7R amplified a 1,274-bp PCR product, larger than expected (Fig. 4), and it was directly sequenced from reference sample 9PA. Locus SC1-7 (GenBank accession MH733246) was $100 \%$ identical to CP004005 (' $\mathrm{Ca}$. L. asiaticus' strain gxpsy), while being 337-bp larger than HQ377374 (' $\mathrm{Ca}$. L. asiaticus' strain UF506). It shares $100 \%$ identity to amino acids 1 to 72 from SC1_gp105 (partial match) and $100 \%$ identity to the complete SC1_gp110.

Because amplification with SC2-type PCR markers was only partial, we attempted to obtain, by PCR, the SC2_SC1 prophage junction, because it is expected to occur in form I prophages in citrus (Zhang et al. 2011). Primers SC2-8F and SC1-1R anneal a single position in $\mathrm{SC} 2$ and $\mathrm{SC} 1$ prophages, respectively. From $\mathrm{SC} 2-8 \mathrm{~F}$ to

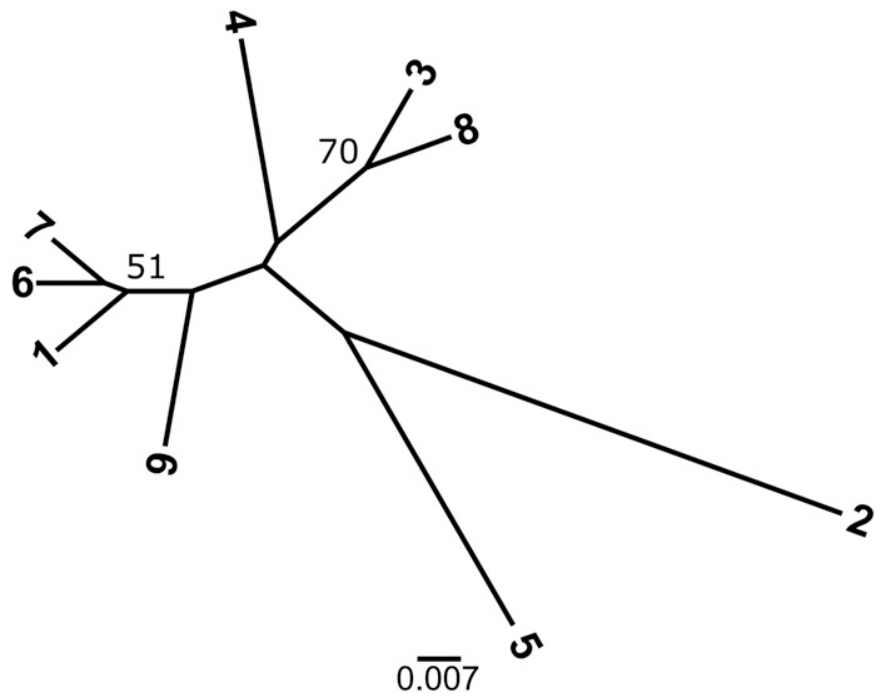

Fig. 1. Dendrogram based on the unweighted pair group method with arithmetic mean estimated with Nei's distance showing the relationship between populations of 'Candidatus Liberibacter asiaticus' from Brazil, estimated from short tandem repeats for three loci. Bootstrap values above 50 are shown. Numbers 1 to 9 at branches represent ' $\mathrm{Ca}$. L. asiaticus' populations in plant samples 2010 to 2015 ; namely, population $1=$ central region, population $2=$ northwest region, population $3=$ north region, population $4=$ southwest region, population $5=$ south region, and population $6=$ Mogi Mirim Farm; and ' $\mathrm{Ca}$. L. asiaticus' in psyllid samples 2015 ; namely, population $7=$ central region, population $8=$ north region, and population $9=$ south region. 
the end of prophage SC2, there are 4,197 bp (HQ377373) whereas, from the beginning of SC1 to the annealing position of SC1-1R, there are 5,898 bp (HQ377372). In form I, SC1 prophage is in tandem with prophage SC2, and a PCR product of primers SC2-8F and SC1-1R would have approximately $10,095 \mathrm{bp}$. Instead of an expected 10-kbp PCR product, as described for UF506 (HQ377374) (Zhang et al. 2011), a 6.1-kbp product was obtained from reference samples (Fig. 5), and the sequence from a 6.1-kbp PCR product from ' $C a$. L. asiaticus' strain 9PA was obtained (GenBank accession
MH733245). This 6,108-bp sequence encodes for seven ORFs highly related to SC2 (type 2) and SC1 (type 1) prophages from UF506 (Table 7). However, gene synteny was only partial between strains 9PA and UF506 (Fig. 6), with the identification of an inversion locus. The 5' ORF SC2_gp230 and 3' ORF SC1_gp035 were similar among 9PA, UF506, and other ' $\mathrm{Ca}$. L. asiaticus' sequenced genomes. Following SC2_gp230, three ORFs are missing in ' $C a$. L. asiaticus' strain 9PA: SC2_gp240, SC2_gp245, and SC2_gp250. Next is SC1_gp235, which in UF506 is

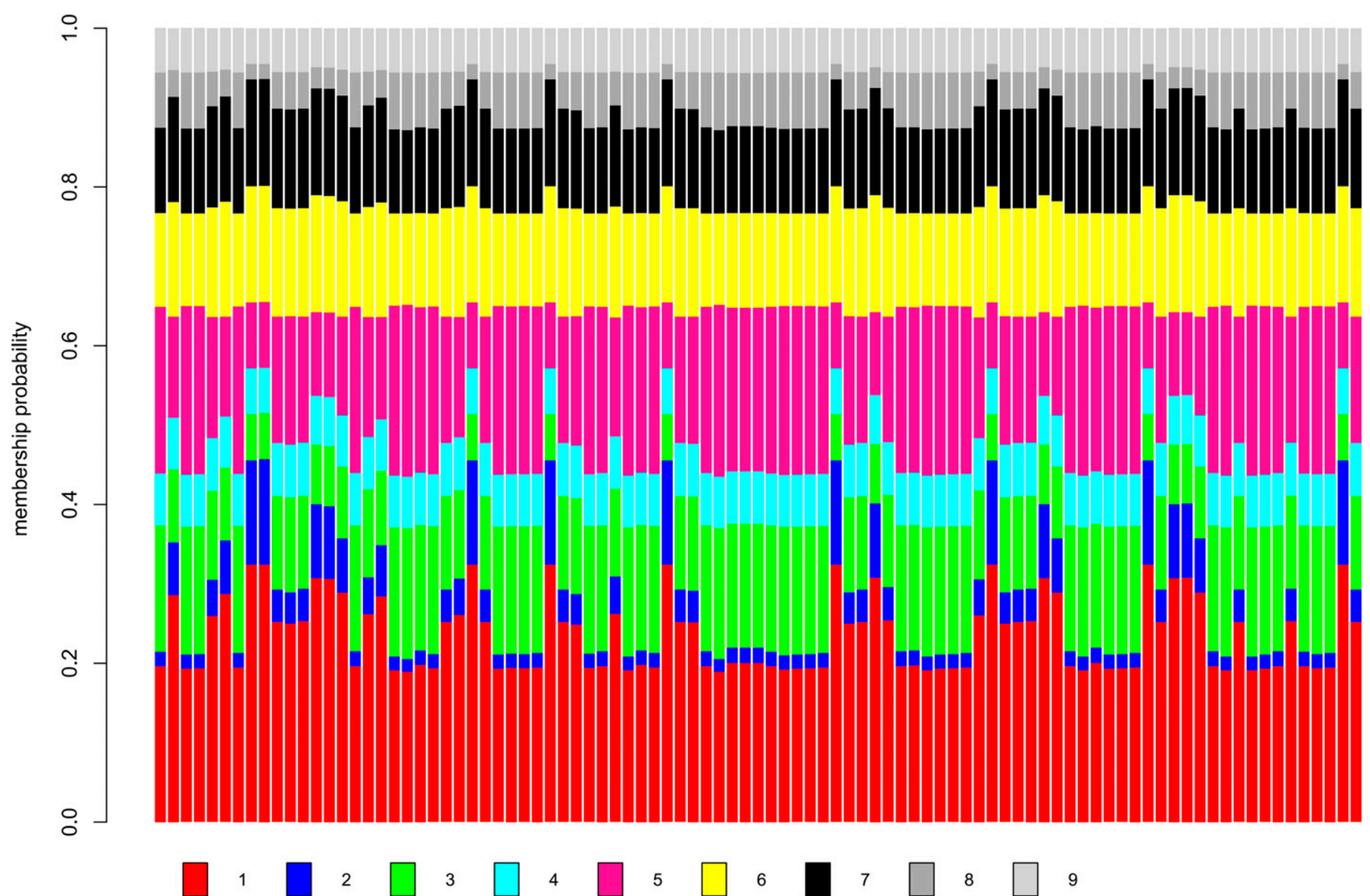

Fig. 2. Genetic structure based on the variation of short tandem repeats for three loci in the genome of 'Candidatus Liberibacter asiaticus' strains from Brazil. Bar plots of discriminant analysis of principal components generated for the clone-corrected dataset $(n=93)$, showing individuals' assignments to genetic clusters. ' $C a$. L. asiaticus' populations are color coded and indicated by numbers 1 to 9. ' $C a$. L. asiaticus' populations in plant samples 2010 to 2015 ; namely, population $1=$ central region, population $2=$ northwest region, population $3=$ north region, population $4=$ southwest region, population $5=$ south region, and population $6=$ Mogi Mirim Farm; and 'Ca. L. asiaticus' in psyllid samples 2015; namely, population $7=$ central region, population $8=$ north region, and population $9=$ south region.

TABLE 6. Analysis of molecular variance performed for short tandem repeats on three loci in the genome of 470 strains of 'Candidatus Liberibacter asiaticus' from Brazil ${ }^{y}$

\begin{tabular}{|c|c|c|c|c|c|c|c|}
\hline Populations $^{\mathrm{z}}$ & df & SS & MS & Variance & Percent & $P$ value & $\Phi_{\mathrm{ST}}$ \\
\hline \multicolumn{8}{|l|}{ All } \\
\hline Among populations & 8 & 13.467 & 1.683 & 0 & 0 & $\ldots$ & $\ldots$ \\
\hline Within populations & 461 & 924.033 & 2.004 & 2.004 & 100 & $\ldots$ & $\ldots$ \\
\hline \multicolumn{8}{|l|}{ Regions } \\
\hline Among regions & 4 & 5.579 & 1.395 & 0.000 & 0 & $\ldots$ & $\ldots$ \\
\hline Within regions & 465 & 931.921 & 2.004 & 2.004 & 100 & $\ldots$ & $\ldots$ \\
\hline Total & 469 & 937.500 & & 2.004 & 100 & $0.616 \mathrm{~ns}$ & -0.004 \\
\hline Within populations & 375 & 735.966 & 1.963 & 1.960 & 99 & $\ldots$ & $\ldots$ \\
\hline Total & 376 & 743.061 & & 1.989 & 100 & $0.015^{*}$ & 0.042 \\
\hline
\end{tabular}

${ }^{\mathrm{y}}$ Abbreviations and symbols: $\mathrm{df}=$ degrees of freedom, $\mathrm{SS}=$ sum of squares, $\mathrm{MS}=$ mean squares, $\mathrm{ns}=$ not significant, $\Phi_{\mathrm{ST}}=\Phi$ statistic estimates, and an asterisk (*) indicates significant at $5 \%$ probability.

${ }^{\mathrm{z}}$ All $=$ all populations, Regions = among regions (center, north, northwest, south, and southwest), and Temporal = 2010 and 2015. 
located at the $3^{\prime}$ extremity of SC1 (not shown in Figure 6). ORFs SC1_gp005 and SC1_gp010 are identical in 9PA and UF506, whereas SC1_gp015 is missing. Next, ORFs SC1_gp025 (hyvI), SC1_gp030, and SC1_gp035 are in the same orientation as in UF506. ORF 4 from ' $C a$. L. asiaticus' strain 9PA is highly similar to SC1_gp010 as well as to SC2_gp255 from ' $\mathrm{Ca}$. L. asiaticus' strain UF506. In ' $\mathrm{Ca}$. L. asiaticus' strain UF506, these ORFs are apart in the SC1/SC2 junction. SC2_gp260 and SC2_gp265 are also missing in this PCR product from 'Ca. L. asiaticus' strain 9PA. Although SC2_gp240 is not located in this PCR product, it was identified in the PCR product SC2-8, indicating another place where gene rearrangement might occur in prophages type 1 or type 2-like from Brazilian ' $\mathrm{Ca}$. L. asiaticus' strains.

PCR with primer set 9PA-1F/9PA-1R was used to show the presence of the inversion locus, amplifying the 567-bp DNA fragment in $97.8 \%$ of the samples from Brazil. In contrast, only 10 of 82 samples (12.2\%) from central Florida clearly amplified with the same set of primers, whereas 72 were negative in PCR (Fig. 6). This primer pair would anneal $39 \mathrm{kbp}$ apart, taking the UF506 genome as a reference and producing no obvious amplification where the inversion locus is not present.

\section{DISCUSSION}

The usefulness of STR analysis previously developed for ' $\mathrm{Ca}$. L. asiaticus' characterization (Chen et al. 2010; Islam et al. 2012; Katoh et al. 2011, 2015; Ma et al. 2014; Matos et al. 2013) was further explored here. Following a scheme developed by Matos et al. (2013), ' $C a$. L. asiaticus' samples were grouped in eight classes in this study whereas, in Florida, only two classes were found, probably as a reflection of sampling size. Although haplotype classes HA and HB were not found in ' $\mathrm{Ca}$. L. asiaticus' samples from Brazil (Matos et al. 2013), we report the occurrence of nine samples with locus VNTR_005 (motif A) with less than 10 repetitions. This characteristic was not previously reported for Brazilian ' $C a$. L. asiaticus' strains (Deng et al. 2014; Islam et al. 2012; Matos et al. 2013), indicating that sample size matters, particularly for a deeper characterization of bacteria strains from a

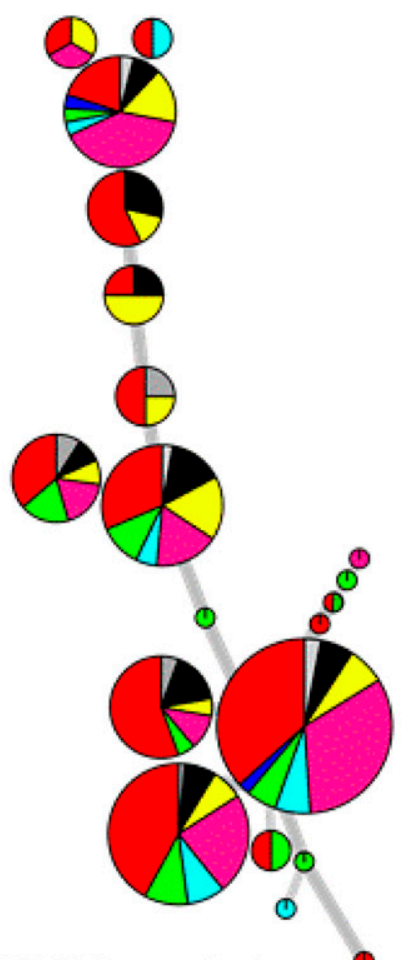

1: $2010 / 2015$ - central

2: $2010 / 2015$ - northwest

3: $2010 / 2015$ - north

4: $2010 / 2015$ - southwest

5: 2010/2015 - south

6: Mogi Mirim Farm - south

7: psyllid/2015 - central

8: psyllid/2015 - north

9: psyllid/2015 - south

$0.167 \quad 0.167$

0.167

0.167

\section{DISTANCE}

Fig. 3. Minimum spanning network and distribution of haplotype genetic variation. Each circle represents a distinct haplotype, in that size of circles is proportional to their frequencies, containing single haplotypes identified in the distinct populations $(n=93)$. 'Candidatus Liberibacter asiaticus' populations are color-coded: ' $\mathrm{Ca}$. L. asiaticus' populations in plant samples 1 to 6 and ' $\mathrm{Ca}$. L. asiaticus' in 2015 psyllid samples populations 7 to 9. 
large crop, as in the case of sweet orange in Brazil. Sweet orange was cultivated in 402,000 ha in 2017, with 191 million sweet orange trees and average HLB incidence of $18.15 \%$ in São Paulo and Minas Gerais States (https://www.fundecitrus.com.br/index.php/ levantamentos/greening). Although 38 haplotypes were found in our analysis, Islam et al. (2012) found 117 haplotypes in a worldwide 'Ca. L. asiaticus' collection, using seven STR loci. A deeper analysis of ' $\mathrm{Ca}$. L. asiaticus' strains from Brazil could be carried out with the use of additional polymorphic loci, with a greater possibility of increasing the haplotype number. Only VNTR_005 or trn1 (Chen et al. 2010; Katoh et al. 2011; Matos et al. 2013) overlaps with the seven STR markers employed by Islam et al. (2012). However, in 'Ca. L. asiaticus' strains from Brazil analyzed by Islam et al. (2012), three of seven loci were monomorphic, rendering four STR loci polymorphic in comparison with three polymorphic STR markers employed in the current study. The common STR locus (VNTR_005, trn 1, or LasSSR-E) is a good indicator of genotypic diversity in global (Islam et al. 2012) and regional (Katoh et al. 2011; Ma et al. 2014) analyses and useful in population comparison (Chen et al. 2010; Deng et al. 2014). However, the use of only this single VNTR for diversity studies would turn our 470 single-peak strains into only 13 haplotypes instead of the 38 found, clearly indicating the importance of the use of a larger number of STR loci. Islam et al. (2012) would have found 5 haplotypes instead of 14 for the 22 samples from Brazil, if this single locus was used instead. The most common repeat number for this locus in ' $\mathrm{Ca}$. L. asiaticus' strains from Brazil is 15 repetitions, with varying incidences according to the study and population size: $100 \%$ for two strains (Matos et al. 2013), $68.1 \%$ among 22 strains (Islam et al. 2012), 66.7\% among 85 strains (Deng et al. 2014), and $58.3 \%$ in the current work.

For all population subsets, haplotype class HE was predominant. Samples from the Mogi Mirim farm were collected from a wellmanaged sweet orange orchard, subjected to HLB-affected tree eradication in a 3-month interval. In this way, symptomatic trees were found soon after symptoms became visible. Thus, ' $\mathrm{Ca}$. L. asiaticus' samples found were, in fact, from recent primary infections and found to be the less variable population subset in relation to haplotype classes and in number of samples with double peaks. This population had, however, one of the highest genotypic diversities. In contrast, ' $\mathrm{Ca}$. L. asiaticus' from population 2010-11 collected by growers in the orchards and from the HLB survey population in 2015 had higher haplotypic variability. These populations were also associated with the occurrence of double peaks at certain STR loci. Management and multiple infections may have an effect on such analysis.

STR analysis revealed double peaks for loci VNTR_005, VNTR_002, and VNTR_077 in 97 samples (16.9\%). Reference samples and the ACP population had the highest percentage of double peaks (33.3 and $24.6 \%$, respectively). The same was observed in China (Ma et al. 2014) and in a few ' $\mathrm{Ca}$. L. asiaticus' strains from plant samples in Japan (Katoh et al. 2011). The population from 2010-11 subset had only $8.5 \%$ when compared with $20.7 \%$ of the population from 2015 . The number of STRs in ' $C a$. L. asiaticus' strains after psyllid acquisition and transmission was reported as varying (Katoh et al. 2015) and its role on

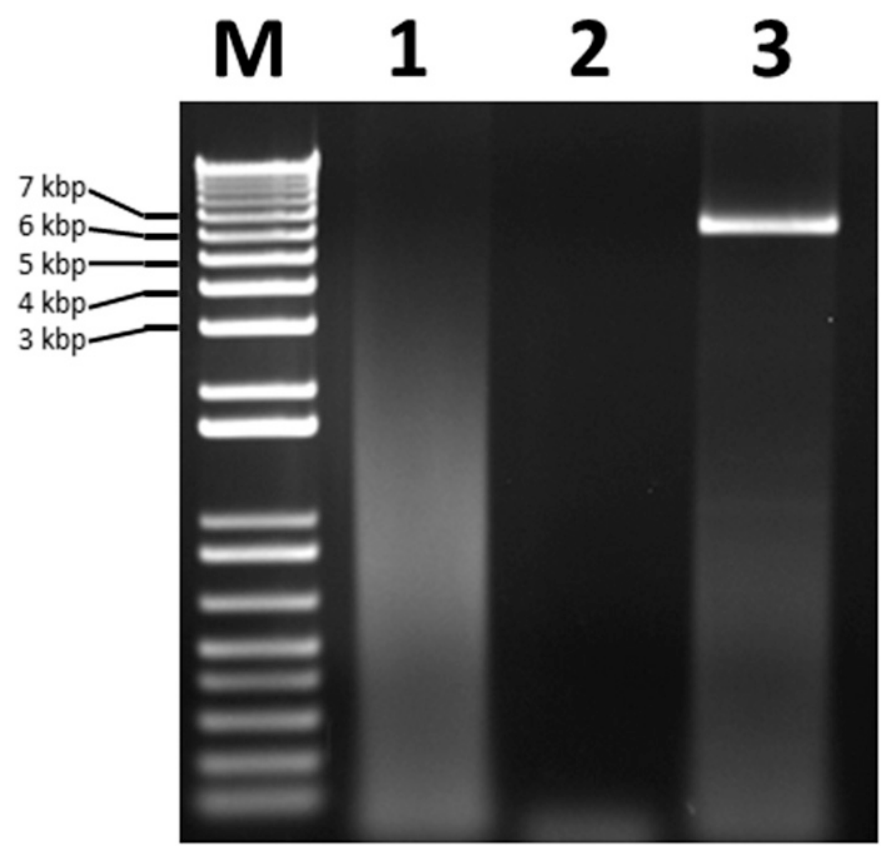

Fig. 5. Polymerase chain reaction amplification using primers $\mathrm{SC} 2-8 \mathrm{~F}$ and SC1-1R for prophage SC2_SC1 junction form I. Lane 1 = healthy plant, lane 2 = nontemplate control, and lane $3=$ 'Candidatus Liberibacter asiaticus' DNA from strain 9PA. Lane $\mathrm{M}=1-\mathrm{kb}$ plus marker from Invitrogen.

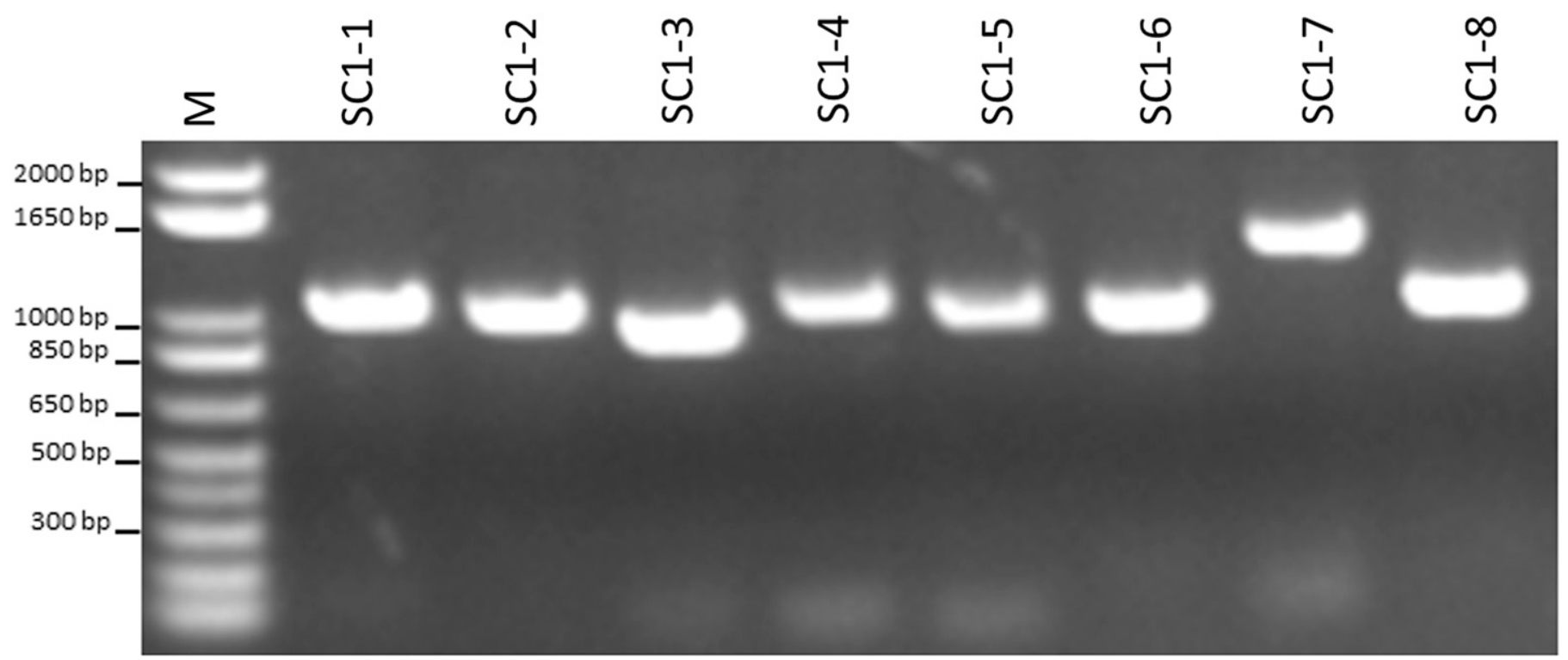

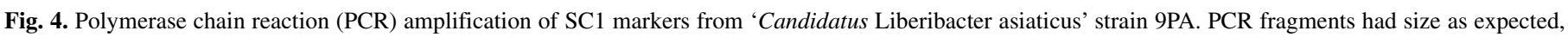

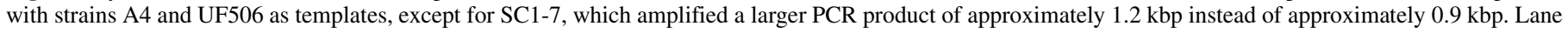
$\mathrm{M}=1-\mathrm{kb}$ plus marker from Invitrogen. 
' $\mathrm{Ca}$. L. asiaticus' strain diversity in field conditions should be evaluated. The occurrence of double peaks (more than one allele) might (i) indicate the presence of more than a single ' $\mathrm{Ca}$. L. asiaticus' genotype or ' $\mathrm{Ca}$. L. asiaticus' strain resulting from different inoculations by the psyllids or (ii) represent the occurrence of the DNA slippage mechanism and the generation of a new haplotype, present along the original haplotype of ' $\mathrm{Ca}$. L. asiaticus' (Chen et al. 2010; van Belkum et al. 2007).

STR analysis supports low genetic diversity observed in the ' $\mathrm{Ca}$. L. asiaticus' population from SPS strains. Cluster analysis agreed with results found by Islam et al. (2012), where a similar genetic makeup among strains was found in Brazilian samples. This fact suggests homogeneity in Brazilian samples, with only one probable introduction from Asia (Islam et al. 2012), in contrast to a probable double introduction in Florida (Chen et al. 2010; Islam et al. 2012).

A lower genetic diversity is also observed for Brazilian ' $\mathrm{Ca}$. L. asiaticus' strains when compared with Japanese strains (Katoh et al. 2011). Although citrus in Japan was recently colonized by ' $\mathrm{Ca}$. L. asiaticus' in a time scale comparable with that in Brazil, the proximity of countries where ' $\mathrm{Ca}$. L. asiaticus' has been found for centuries, such as China (Bové 2006), may reflect differences in diversity. The influence of the environment on the epidemiological

TABLE 7. Open reading frame (ORF) prediction and protein identification for the 6.1-kbp prophage amplicon obtained from 'Candidatus Liberibacter asiaticus' strain 9PA using primers SC2-8F and SC1-1R (MH733245) ${ }^{\mathrm{z}}$

\begin{tabular}{|c|c|c|c|c|c|}
\hline ORF & Strand & Size (aa) & Protein BLAST & E value & Putative function \\
\hline 1 & + & $66\left(3^{\prime}\right.$ partial $)$ & SC2_gp230 (190 aa) [KPG62725.1] & $1 e-38$ & Guanylate kinase \\
\hline 2 & + & 116 & SC1_gp235 (123 aa) [ADV02628.1] & $9 e-73$ & Guanylate kinase \\
\hline 3 & - & 157 & SC1_gp005 (157 aa) [ADV02629.1] & $5 e-104$ & Hypothetical protein \\
\hline \multirow[t]{3}{*}{4} & - & 90 & SC1_gp010 (90 aa) [ADV02630.1] & $4 e-58$ & Hypothetical protein \\
\hline & $\cdots$ & $\cdots$ & SC2_gp010 (90 aa) [ADV02588.1] & $2 \mathrm{e}-57$ & Hypothetical protein \\
\hline & $\ldots$ & $\ldots$ & SC2_gp255 (90 aa) [ADV02584.1] & $1 e-54$ & Colicin immunity protein \\
\hline 5 & - & 646 & AEK98981 (hyvI) (646 aa) & 0.0 & Autotransporter \\
\hline \multirow[t]{2}{*}{6} & - & 864 & WSI_05615 (864 aa) [AGH17480] & 0.0 & Hypothetical protein \\
\hline & $\ldots$ & $\ldots$ & SC1_gp030 (864 aa) [ADV02633.1] & 0.0 & Hypothetical protein \\
\hline 7 & - & $37\left(3^{\prime}\right.$ partial $)$ & SC1_gp035 (478 aa) [ADV02634.1] & $2 \mathrm{e}-32$ & Hypothetical protein \\
\hline
\end{tabular}

z Abbreviation: aa $=$ amino acids.

A

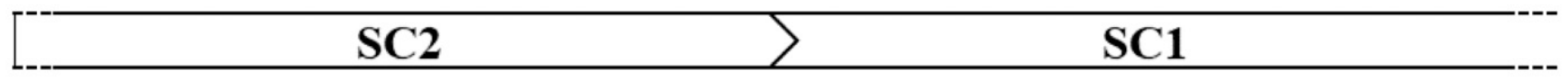

UF506
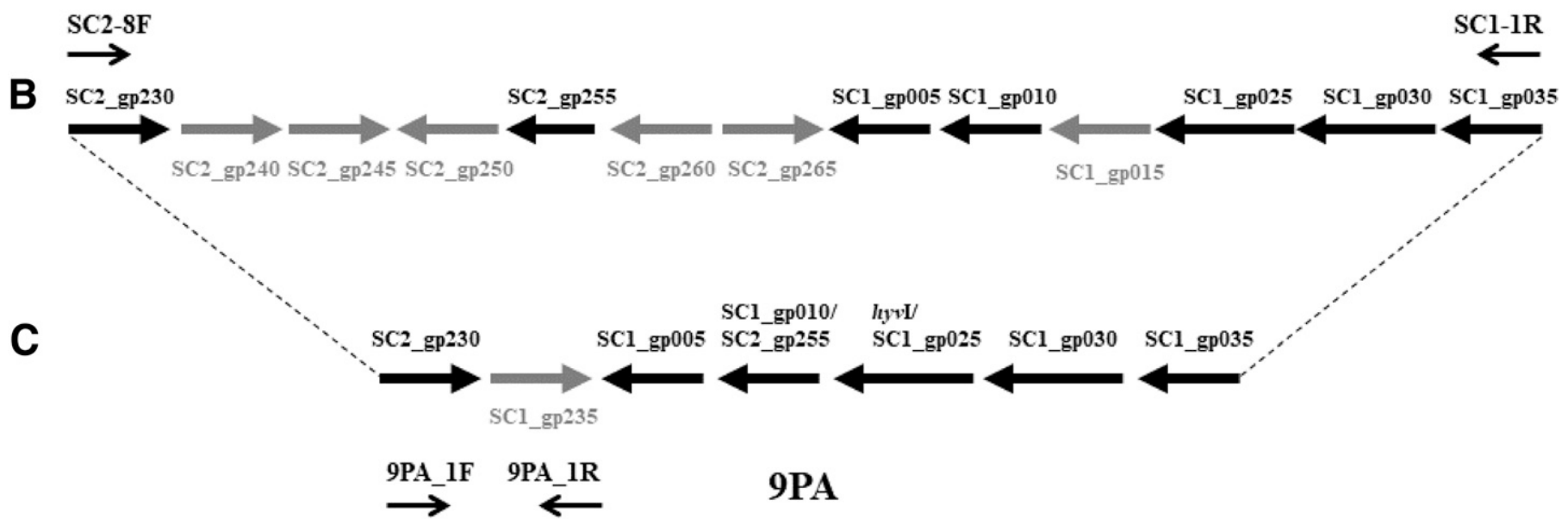

D

$\begin{array}{llllllllllllllllllll}M & 1 & 2 & 3 & 4 & 5 & 6 & 7 & 8 & 9 & 10 & 11 & 12 & 13 & 14 & 15 & 16 & 17 & 18 & 19\end{array}$

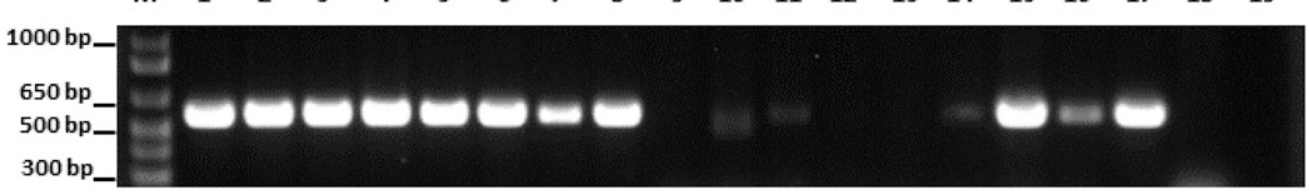

Fig. 6. Prophages SC2/SC1 form I organization in 'Candidatus Liberibacter asiaticus'. A, Prophage junction and B, open reading frame (ORF) found between annealing sites of primers SC2-8F (forward) and SC1-1R (reverse) in ' $\mathrm{Ca}$. L. asiaticus' UF506, with ORF names according to Zhang et al. (2011). Black arrows are ORFs common between UF506 and Brazilian strain 9PA, while gray arrows are ORFs not present in the polymerase chain reaction (PCR) fragment from ' $\mathrm{Ca}$. L. asiaticus' strain 9PA. C, Correspondent ORFs found in ' $\mathrm{Ca}$. L. asiaticus' strain 9PA, following nomenclature from the highest blast hit. Gray arrow indicates a single ORF found in strain 9PA in a position divergent from UF506. D, Detection of inversion locus between prophages SC2/SC1 in ' $\mathrm{Ca}$. L. asiaticus' samples from Brazil and the United States. PCR results using primers 9PA-1F and 9PA-1R. Lanes 1 to $4=$ ' $C a$. L. asiaticus' strains from the 2015 subset, Brazil; lanes 5 to $8=$ ' $\mathrm{Ca}$. L. asiaticus' strains from the single farm in Mogi Mirim, São Paulo, Brazil; lanes 9 to $16=$ ' $\mathrm{Ca}$. L. asiaticus' strains from Central Florida, 2016 ; lane $17=$ 'Ca. L. asiaticus' strain 9PA (Brazil); lane $18=$ healthy plant; and lane $19=$ nontemplate control. Lane M is $1 \mathrm{kbp}$ plus marker from Invitrogen. 
progress (north to south comparison) of HLB in Brazil (Lopes et al. 2017) does not necessarily reflect differences in fitness among ' $\mathrm{Ca}$. L. asiaticus' strains not observed in the current analysis. As of today, there is no link between genetic diversity and pathogenicity, due to the limited knowledge we have regarding ' $\mathrm{Ca}$. L. asiaticus' virulence mechanisms.

' $C a$. L. asiaticus' strains from Brazil were of genotype Term-A, following the same result previously observed but being significantly different from those in the United States and China (Deng et al. 2014). The high number of ' $\mathrm{Ca}$. L. asiaticus' strains employed in our work precludes sample size limitations to point out the absence of genotypes 3 and 4 (Deng et al. 2014) and reveals the low incidence (1.21\%) of the genotype 1 (Deng et al. 2014).

Prophages are present in most sequenced Liberibacter genomes known thus far, though the origin of prophages from plantassociated Liberibacters and from Liberibacter crescens is not homologous, despite their close relatedness (Frampton et al. 2018; Leonard et al. 2012; Wulff et al. 2014). Due to this omnipresence, the characterization of the prophage occurrence and variability was assessed. PCR markers for both prophages type 1 and 2 were present in $96 \%$ of the 2015 ' $\mathrm{Ca}$. L. asiaticus' population, whereas partial types 1 and 2 were found in only $4 \%$ of it. Although PCR markers for type 1 were mostly present, for type 2 , only one PCR marker was frequently found, indicating either partial presence of this prophage or sequence variation that precludes PCR amplification and detection. Because PCR amplification is sequence dependent and although PCR is useful to screen large populations, variable regions such as those from prophages are better analyzed by sequenceindependent methods such as high-throughput sequencing or nextgeneration sequencing. Sequencing of ' $\mathrm{Ca}$. L. asiaticus' allowed characterization of a ' $\mathrm{Ca}$. L. asiaticus' strain without prophage as Ishi-1 (Katoh et al. 2014), with a single prophage as strain A4 (Zheng et al. 2014), two prophages as ' $\mathrm{Ca}$. L. asiaticus' strains psy62, UF506, and gxpsy (Duan et al. 2009; Lin et al. 2013; Zhang et al. 2011), and also three prophages (Zheng et al. 2018). The two present prophages, SC1 in a similar way to UF506 (SC1-type) and SC-2 only partially found (SC2-like), are the predominant genotypes found in Brazil. SC2 harbors peroxidases that may repress plant defense responses (Jain et al. 2015) although, as observed for strain Ishi-1, which lacks prophages, they are not absolutely necessary for ' $\mathrm{Ca}$. L. asiaticus' survival. We have not found ' $\mathrm{Ca}$. L. asiaticus' strains without type 1 and type 2 prophages, which is observed in a percentage ranging from 2.67 to $3.44 \%$ of samples in China (Zheng et al. 2016, 2018).

The presence of prophages SC1-type and SC2-type with an inversion locus coupled with partial deletion in early genes of SC2 in the ' $C a$. L. asiaticus' population from Brazil was found, indicating a different genomic organization than previously reported for other ' $C a$. L. asiaticus' populations and genomes sequenced thus far (SC2-like). Wang et al. (2012) reported a mosaic locus in type 1 prophage among ' $\mathrm{Ca}$. L. asiaticus' strains from China and the United States, with three insertions (72, 109, and 274 bp) in SC1_gp140, allowing typing of the ' $C a$. L. asiaticus' population by PCR. In our case, gene deletion and a unique gene organization allowed typing of the ' $\mathrm{Ca}$. L. asiaticus' population from Brazil. Conversely, Florida samples were mostly negative for this new configuration, although the inversion locus was sometimes present as well. A multilocus deletion in ' $\mathrm{Ca}$. L. asiaticus' was reported by Wang et al. (2015) for ORFs CLIBASIA_05645, CLIBASIA_05650, and CLIBASIA_05655, which are, in fact, ORFs duplicated in both SC1 and SC2 (_gp145, gp150, and _gp160, respectively). In our case, ORF deletion was reported for the type 2-like prophage.

Additional prophages besides SC1 and SC2 were reported by Zhou et al. (2013), while a type 3 prophage, recently described (Zheng et al. 2018), was not screened and should be evaluated in the future in ' $C a$. L. asiaticus' strains from Brazil. ' $C a$. L. asiaticus' strains had PCR product SC1-7F/SC1-7R larger than reported
(Zheng et al. 2016) and more similar to ' $\mathrm{Ca}$. L. asiaticus' from China (gxpsy and YCPsy strains) than to U.S. strain UF506 (Zhang et al. 2011). The presence of multiple PCR sizes for SC1 markers in the ' $\mathrm{Ca}$. L. asiaticus' population was reported before (Wang et al. 2012; Zhou et al. 2011) and was of limited occurrence in our sampling. Collectively, a ' $\mathrm{Ca}$. L. asiaticus' population with limited variability is found in Brazil, which is typical for a clonal population recently introduced into a new geographical location and perhaps kept with limited variation due to both avoidance of new introductions and constant tree eradication carried out by growers as part of HLB management.

\section{ACKNOWLEDGMENTS}

We thank L. Peña for critically reviewing the manuscript and $O . Z$. Zanardi and H. X. L Volpi for running Fisher's test.

\section{LITERATURE CITED}

Ayres, M., Jr., Ayres, D. L., and Santos, A. S. S. 2007. BioEstat 5.0. Aplicações estatísticas nas áreas das ciências bio-médicas. Universidade Federal do Pará. Belém, PA, Brazil.

Bastianel, C., Garnier-Semancik, M., Renaudin, J., Bové, J. M., and Eveillard, S. 2005. Diversity of "Candidatus Liberibacter asiaticus," based on the omp gene sequence. Appl. Environ. Microbiol. 71:6473-6478.

Besemer, J., and Borodovsky, M. 1999. Heuristic approach to deriving models for gene finding. Nucleic Acids Res. 27:3911-3920.

Bové, J. M. 2006. A destructive, newly-emerging, century-old disease of citrus. J. Plant Pathol. 88:7-37.

Bové, J. M. 2012. Huanglongbing and the future of citrus in São Paulo state, Brazil. J. Plant Pathol. 94:465-467.

Bruvo, R., Michiels, N. K., D’Souza, T. G., and Schulenburg, H. 2004. A simple method for the calculation of microsatellite genotype distances irrespective of ploidy level. Mol. Ecol. 13:2101-2106.

Chen, J., Deng, X., Sun, X., Jones, D., Irey, M., and Civerolo, E. 2010. Guangdong and Florida populations of 'Candidatus Liberibacter asiaticus' distinguished by a genomic locus with short tandem repeats. Phytopathology 100:567-572.

Chen, J., Pu, X., Deng, X., Liu, S., Li, H., and Civerolo, E. 2009. A phytoplasma related to 'Candidatus Phytoplasma asteris' detected in citrus showing huanglongbing (yellow shoot disease) symptoms in Guangdong, P. R.China. Phytopathology 99:236-242.

Coletta-Filho, H. D., Targon, M. L. P. N., Takita, M. A., De Negri, J. D., Pompeu, J. J., and Machado, M. A. 2004. First report of the causal agent of Huanglongbing ("Candidatus Liberibacter asiaticus") in Brazil. Plant Dis. $88: 1382$.

da Graça, J. V., Douhan, G. W., Halbert, S. E., Keremane, M. L., Lee, R. F., Vidalakis, G., and Zhao, H. 2016. Huanglongbing: An overview of a complex pathosystem ravaging the world's citrus. J. Integr. Plant Biol. 58: 373-387.

Deng, X., Lopes, S., Wang, X., Sun, X., Jones, D., Irey, M., Civerolo, E., and Chen, J. 2014. Characterization of "Candidatus Liberibacter asiaticus" populations by double-locus analyses. Curr. Microbiol. 69:554-560.

Dray, S., and Dufour, A. B. 2007. The ade4 package: Implementing the duality diagram for ecologists. J. Stat. Softw. 22:1-20.

Duan, Y., Zhou, L., Hall, D. G., Li, W., Doddapaneni, H., Lin, H., Liu, L., Vahling, C. M., Gabriel, D. W., Williams, K. P., Dickerman, A., Sun, Y., and Gottwald, T. 2009. Complete Genome Sequence of Citrus Huanglongbing bacterium, "Candidatus Liberibacter asiaticus" obtained through metagenomics. Mol. Plant-Microbe Interact. 22:1011-1020.

Frampton, R. A., Thompson, S. M., Kalamorz, F., David, C., Addison, S. M., and Smith, G. R. 2018. Draft genome sequence of a "Candidatus Liberibacter europaeus" strain assembled from broom psyllids (Arytainilla spartiophila) from New Zealand. Genome Announce. 6:e0043018.

Fundecitrus. 2018. Levantamento de Greening/HLB. https://www.fundecitrus. com.br/levantamentos/greening

Gétaz, M., Krijger, M., Rezzonico, F., Smits, T. H. M., Van der Wolf, J. M., and Pothier, J. F. 2018. Genome-based population structure analysis of the strawberry plant pathogen Xanthomonas fragariae reveals two distinct groups that evolved independently before its species description. Microb. Genom. 4.

Hocquellet, A., Toorawa, P., Bové, J. M., and Garnier, M. 1999. Detection and identification of the two Candidatus Liberobacter species associated with citrus huanglongbing by PCR amplification of ribosomal protein genes of the $\beta$ operon. Mol. Cell. Probes 13:373-379.

Islam, M. S., Glynn, J. M., Bai, Y., Duan, Y. P., Coletta-Filho, H. D., Kuruba, G., Civerolo, E. L., and Lin, H. 2012. Multilocus microsatellite analysis of 
'Candidatus Liberibacter asiaticus' associated with citrus Huanglongbing worldwide. BMC Microbiol. 12:39.

Jain, M., Fleites, L. A., and Gabriel, D. W. 2015. Prophage-encoded peroxidase in 'Candidatus Liberibacter asiaticus' is a secreted effector that suppresses plant defenses. Mol. Plant-Microbe Interact. 28:1330-1337.

Jombart, T., and Ahmed, I. 2011. Adegenet 1.3-1: New tools for the analysis of genome-wide SNP data. Bioinformatics 27:3070-3071.

Jombart, T., Devillard, S., and Balloux, F. 2010. Discriminant analysis of principal components: A new method for the analysis of genetically structured populations. BMC Genet. 11:94.

Kamvar, Z. N., Tabima, J. F., and Grünwald, N. J. 2014. Poppr: An R package for genetic analysis of populations with clonal, partially clonal, and/or sexual reproduction. PeerJ 2:e281.

Katoh, H., Inoue, H., and Iwanami, T. 2015. Changes in variable number of tandem repeats in 'Candidatus Liberibacter asiaticus' through insect transmission. PLoS One 10:e0138699.

Katoh, H., Miyata, S., Inoue, H., and Iwanami, T. 2014. Unique features of a Japanese 'Candidatus Liberibacter asiaticus' strain revealed by whole genome sequencing. PLoS One 9:e106109.

Katoh, H., Subandiyah, S., Tomimura, K., Okuda, M., Su, H. J., and Iwanami, T. 2011. Differentiation of "Candidatus Liberibacter asiaticus" isolates by variable-number tandem-repeat analysis. Appl. Environ. Microbiol. 77: 1910-1917.

Kumar, S., Stecher, G., and Tamura, K. 2016. MEGA7: Molecular Evolutionary Genetics Analysis Version 7.0 for Bigger Datasets. Mol. Biol. Evol. 33:1870-1874

Leonard, M. T., Fagen, J. R., Davis-Richardson, A. G., Davis, M. J., and Triplett, E. W. 2012. Complete genome sequence of Liberibacter crescens BT-1. Stand. Genomic Sci. 7:271-283.

Li, W., Hartung, J. S., and Levy, L. 2006. Quantitative real-time PCR detection and quantification of Candidatus Liberibacter species associated with citrus huanglongbing. J. Microbiol. Methods 66:104-115.

Lin, H., Han, C. S., Liu, B., Lou, B., Bai, X., Deng, C., Civerolo, E. L., and Gupta, G. 2013. Complete genome sequence of a Chinese strain of "Candidatus Liberibacter asiaticus". Genome Announce. 1:e00184-e13.

Lin, H., Islam, M. S., La Rosa, J. C. C., Civerolo, E. L., and Groves, R. L. 2015. Population structure of Xylella fastidiosa associated with almond leaf scorch disease in the San Joaquin Valley of California. Phytopathology 105: 825-832.

Liu, R. R., Zhang, P., Pu, X., Xing, X., Chen, J., and Deng, X. 2011. Analysis of a prophage gene frequency revealed population variation of 'Candidatus Liberibacter asiaticus' from two citrus-growing provinces in China. Plant Dis. 95:431-435

Lopes, S. A., Luiz, F. Q. B. F., Oliveira, H. T., Cifuentes-Arenas, J., and Raiol, L. L., Jr. 2017. Seasonal variation of 'Candidatus Liberibacter asiaticus' titers in new shoots of citrus in distinct environments. Plant Dis. 101: 583-590.

Ma, W., Liang, M., Guan, L., Xu, M., Wen, X., Deng, X., and Chen, J. 2014. Population structures of 'Candidatus Liberibacter asiaticus' in southern China. Phytopathology 104:158-162.

Matos, L. A., Hilf, M. E., Chen, J., and Folimonova, S. Y. 2013. Validation of 'variable number of tandem repeat'-based approach for examination of 'Candidatus Liberibacter asiaticus' diversity and its applications for the analysis of the pathogen populations in the areas of recent introduction. PLoS One 8:e78994.

Murray, M. G., and Thompson, W. F. 1980. Rapid isolation of high molecular weight plant DNA. Nucleic Acids Res. 8:4321-4326.

Nei, M. 1972. Genetic distances between populations. Am. Nat. 106:283-292.

Peakall, R., and Smouse, P. E. 2012. GenAlEx 6.5: Genetic analysis in Excel. Population genetic software for teaching and research-An update. Bioinformatics 28:2537-2539.

Pérez-Otero, R., Mansilla, J. P., and Del Estal, P. 2015. Detección de la psila africana de los cítricos, Trioza erytreae (Del Guercio, 1918) (Hemiptera: Psylloidea: Triozidae), en la Península Ibérica. Arq. Entomol. 13:119-122.

Pietersen, G., Arrebola, E., Breytenbach, J. H. J., Korsten, L., le Roux, H. F., la Grange, H., Lopes, S. A., Meyer, J. B., Pretorius, M. C., Schwerdtfeger, M., van Vuuren, S. P., and Yamamoto, P. 2010. A survey for 'Candidatus Liberibacter' species in south Africa confirms the presence of only ' $\mathrm{Ca}$. L. africanus' in commercial citrus. Plant Dis. 94:244-249.

R Core Team. 2015. R: A Language and Environment for Statistical Computing. R Foundation for Statistical Computing, Vienna, Austria.

Roberts, R., Cook, G., Grout, T. G., Khamis, F., Rwomushana, I., Nderitu, P. W., Seguni, Z., Materu, C. L., Steyn, C., Pietersen, G., Ekesi, S., and le Roux, H. F. 2017. Resolution of the identity of 'Candidatus Liberibacter' species from Huanglongbing-affected citrus in east Africa. Plant Dis. 101: 1481-1488.
Roberts, R., Steenkamp, E. T., and Pietersen, G. 2015. Three novel lineages of 'Candidatus Liberibacter africanus' associated with native rutaceous hosts of Trioza erytreae in South Africa. Int. J. Syst. Evol. Microbiol. 65:723-731.

Roullier, C., Benoit, L., McKey, D. B., and Lebot, V. 2013. Historical collections reveal patterns of diffusion of sweet potato in Oceania obscured by modern plant movements and recombination. Proc. Natl. Acad. Sci. U.S.A. 110:2205-2210.

Sievers, F., and Higgins, D. G. 2018. Clustal Omega for making accurate alignments of many protein sciences. Protein Sci. 27:135-145.

Szpiech, Z. A., and Rosenberg, N. A. 2011. On the size distribution of private microsatellite alleles. Theor. Popul. Biol. 80:100-113.

Teixeira, D. C., Danet, J. L., Eveillard, S., Martins, E. C., de Jesus, W. C., Jr., Yamamoto, P. T., Lopes, S. A., Bassanezi, R. B., Ayres, A. J., Saillard, C., and Bové, J. M. 2005. Citrus huanglongbing in São Paulo State, Brazil: PCR detection of the 'Candidatus' Liberibacter species associated with the disease. Mol. Cell. Probes 19:173-179.

Teixeira, D. C., Wulff, N. A., Martins, E. C., Kitajima, E. W., Bassanezi, R., Ayres, A. J., Eveillard, S., Saillard, C., and Bové, J. M. 2008. A phytoplasma closely related to the pigeon pea witches'-broom phytoplasma (16Sr IX) is associated with citrus huanglongbing symptoms in the state of São Paulo, Brazil. Phytopathology 98:977-984.

Tomimura, K., Miyata, S. I., Furuya, N., Kubota, K., Okuda, M., Subandiyah, S., Hung, T. H., Su, H. J., and Iwanami, T. 2009. Evaluation of genetic diversity among 'Candidatus Liberibacter asiaticus' isolates collected in southeast Asia. Phytopathology 99:1062-1069.

Tyler, H. L., Roesch, L. F. W., Gowda, S., Dawson, W. O., and Triplett, E. W. 2009. Confirmation of the sequence of 'Candidatus Liberibacter asiaticus' and assessment of microbial diversity in huanglongbing-infected citrus phloem using a metagenomic approach. Mol. Plant-Microbe Interact. 22: 1624-1634.

van Belkum, A., Tassios, P. T., Dijkshoorn, L., Haeggman, S., Cookson, B., Fry, N. K., Fussing, V., Green, J., Feil, E., Gerner-Smidt, P., Brisse, S., and Struelens, M. 2007. Guidelines for the validation and application of typing methods for use in bacterial epidemiology. Clin. Microbiol. Infect. 13:1-46.

Wang, X., Su, H., Huang, L., Deng, X., Chen, J., Zhou, C., and Li, Z. 2015. Identification of a novel 1033-nucleotide deletion polymorphism in the prophage region of 'Candidatus Liberibacter asiaticus': Potential applications for bacterial epidemiology. J. Phytopathol. 163:681-685.

Wang, X., Tan, J., Bai, Z., Su, H., Deng, X., Li, Z., Zhou, C., and Chen, J. 2013. Detection and characterization of miniature inverted-repeat transposable elements in "Candidatus Liberibacter asiaticus". J. Bacteriol. 195: 3979-3986.

Wang, X., Zhou, C., Deng, X., Su, H., and Chen, J. 2012. Molecular characterization of a mosaic locus in the genome of 'Candidatus Liberibacter asiaticus'. BMC Microbiol. 12:18.

Wulff, N. A., Fassini, C. G., Marques, V. V., Martins, E. C., Coletti, D. A. B., Teixeira, D. C., Sanches, M. M., and Bové, J. M. 2019. Molecular characterization and detection of 16SrIII group phytoplasma associated with Huanglongbing symptoms. Phytopathology. 109:366-374.

Wulff, N. A., Zhang, S., Setubal, J., Almeida, N. F., Martins, E. C., Harakava, R., Kumar, D., Rangel, L. T., Foissac, X., Bové, J. M., and Gabriel, D. W. 2014. The complete genome sequence of 'Candidatus Liberibacter americanus', associated with Citrus Huanglongbing. Mol. Plant-Microbe Interact. 27:163-176.

Zhang, S., Flores-Cruz, Z., Zhou, L., Kang, B. H., Fleites, L. A., Gooch, M. D., Wulff, N. A., Davis, M. J., Duan, Y. P., and Gabriel, D. W. 2011. ' $C a$. Liberibacter asiaticus' carries an excision plasmid prophage and a chromosomally integrated prophage that becomes lytic in plant infections. Mol. Plant-Microbe Interact. 24:458-468.

Zheng, Z., Bao, M., Wu, F., Chen, J., and Deng, X. 2016. Predominance of single prophage carrying a CRISPR/cas system in "Candidatus Liberibacter asiaticus" strains in southern China. PLoS One 11:e0146422.

Zheng, Z., Bao, M., Wu, F., Van Horn, C., Chen, J., and Deng, X. 2018. A type 3 prophage of 'Candidatus Liberibacter asiaticus' carrying a restrictionmodification system. Phytopathology 108:454-461.

Zheng, Z., Deng, X., and Chen, J. 2014. Whole-genome sequence of "Candidatus Liberibacter asiaticus" from Guangdong, China. Genome Announce. 2:e00273-e14.

Zhou, L., Powell, C., Li, W., Irey, M., and Duan, Y. 2013. Prophage-mediated dynamics of 'Candidatus Liberibacter asiaticus' populations, the destructive bacterial pathogens of citrus Huanglongbing. PLoS One 8:e82248.

Zhou, L., Powell, C. A., Hoffman, M. T., Li, W., Fan, G., Liu, B., Lin, H., and Duan, Y. 2011. Diversity and plasticity of the intracellular plant pathogen and insect symbiont "Candidatus Liberibacter asiaticus" as revealed by hypervariable prophage genes with intragenic tandem repeats. Appl. Environ. Microbiol. 77:6663-6673. 